\title{
SÍNTESE E CARACTERIZAÇÃO DE NANOFIBRAS DE C/BiVO VIA CARBONIZAÇÃO DO POLÍMERO PVA: UM POTENCIAL MATERIAL FOTOELETROCATALÍTICO
}

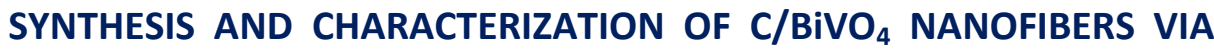 PVA POLYMER CARBONIZATION: A POTENTIAL PHOTOELECTROCATALYTIC MATERIAL}

Ana Carolina Manea Bueno, Bruno Henrique de Santana Gois, Silvio Rainho Teixeira, Deuber Lincon da Silva Agostini, Marcos Fernando de Souza Teixeira, Diego N. David-Parra

Universidade Estadual Paulista - UNESP

E-mail: david.parra@unesp.br

RESUMO - Entidades de todo o mundo estão cada vez mais fortalecendo movimentos em prol de um planeta mais saudável e sustentável. Neste sentido, o intuito deste trabalho é a construção de nanofibras de $\mathrm{C} / \mathrm{BiVO}_{4}$ via eletrofiação e posterior carbonização do polímero PVA. Obtendo assim, nanofibras com elevada área superficial quando comparados aos usais métodos de deposição. Possibilitando maior absorção dos fótons e consequentemente elevando a eficiência fotoeletrocatalítica na conversão energética e degradação de poluentes orgânicos. $\mathrm{O} \mathrm{BiVO}_{4}$ sintetizado foi caracterizado e assim confirmado a formação da fase monoclínica. A técnica de eletrofiação apresentou fibras com diâmetros na escala nanométrica com a presença do carbono, aumentando consideravelmente a área superficial do material, confirmado pelas microscopias óptica e eletrônica de varredura.

Palavras-chave: $\mathrm{BiVO}_{4} ; \mathrm{PVA}$; Nanofibras; Eletrofiação.

ABSTRACT - Entities around the world, are increasingly strengthening movements for a healthier and more sustainable planet. In this sense, the aim of this work is the construction of C/BiVO4 nanofibers via electrospinning and subsequent PVA polymer carbonization. Obtaining thus, nanofibers with high surface area when compared to the usual deposition methods. Enabling greater absorption of photons and consequently increasing the photoelectrocatalytic efficiency in energy conversion and organic pollutants degradation. The synthesized $\mathrm{BiVO}_{4}$ was characterized and thus confirmed the formation of the monoclinic. The electrospinning technique presented fibers with diameters in the nanometric scale with the presence of carbon, considerably increasing 
the surface area of the material, confirmed by optical and scanning electron microscopy.

Keywords: $\mathrm{BiVO}_{4}$; PVA; Nanofibers; Electrospinning. 


\section{INTRODUÇÃO}

O Em 1972 com o pioneiro trabalho de Fujishima (1972), acerca da utilização do material semicondutor $\left(\mathrm{TiO}_{2}\right)$ na área da fotoeletrocatálise, surgiu um novo campo de pesquisa altamente promissor no desenvolvimento de materiais para obtenção de energia limpa a partir da radiação solar e na fotoeletrodegradação de compostos orgânicos poluentes.

Apesar das inúmeras vantagens da aplicação do $\mathrm{TiO}_{2}$, o alto valor de band gap $(3,2 \mathrm{eV})$, diminui drasticamente sua eficiência fotocatalítica devido a absorção do material ocorrer em comprimentos de ondas na região do ultravioleta (UV), região que corresponde apenas $4 \%$ do total do espectro solar Yang (2010).

Na data de 1998, Kudo (1998) realizou um experimento que consistia na incidência de luz na região do visível (Vis) sobre o vanadato de bismuto $\left(\mathrm{BiVO}_{4}\right)$ na presença de íons prata em solução aquosa. Durante o experimento os investigadores observaram a liberação de gás oxigênio. Mostrando assim, uma relação de excitação entre o material semicondutor na região do visível.

Posteriormente, muitos estudos corroboraram o grande potencial fotocatalítico do $\mathrm{BiVO}_{4}$, atribuído diretamente ao seu favorável valor de band gap $(2,4 \mathrm{eV})$, propiciando a absorção do material na região do visível, região a qual corresponde à aproximadamente $43 \%$ do espectro de radiação solar Liu (2015). Uma eficiência 10 vezes maior em relação ao amplamente estudado $\mathrm{TiO}_{2}$. As propriedades fotoeletrocatalíticas do $\mathrm{BiVO}_{4}$ estão intimamente ligadas à suas estruturas cristalinas, sendo possível realizar sua síntese na forma scheelita (monoclínica ou tetragonal) ou zircônia (tetragonal). Tais distinções acarretam uma diferença significativa nos valores de band gap, conforme ilustrado na Figura 1.
Figura 1: Diagrama de bandas das diferentes estruturas cristalinas do $\mathrm{BiVO}_{4}$ e seus respectivos valores de band gap e comprimento de onda de absorção $(\lambda)$.
Zircônia (Tetragonal)

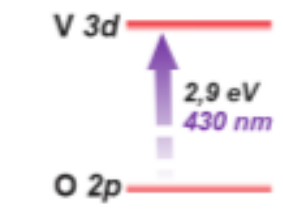

Fonte: Autoria própria
Scheelita (Monoclínica)

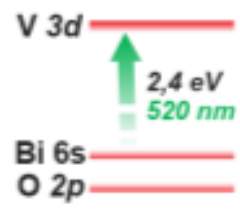

De acordo com a Figura 1, pode-se observar que a fase monoclínica apresenta um menor valor de band gap, sendo a fase de interesse quando sintetizado este material para aplicação em células fotovoltaicas com atividade fotoeletrocatalítica. Segundo Park (2013), a significativa diferença dos valores de band gap entre as fases do $\mathrm{BiVO}_{4}$ ocorre devido a transição eletrônica entre os orbitais. Sendo que na zircônia esta transição ocorre do orbital $2 p$ do oxigênio para o orbital 3d do vanádio, com band gap de 2,9 eV e absorção na região de aproximadamente $430 \mathrm{~nm}$. Já na scheelita a hibridização entre o orbital $6 \mathrm{~s}$ do $\mathrm{Bi}$ e o orbital $2 p$ do $O$, acarreta uma diminuição no band gap $(2,4 \mathrm{eV})$, sendo que nesta fase a transição passa a ocorrer do orbital hibrido para o orbital $3 \mathrm{~d}$ do $\mathrm{V}$, propiciando a absorção na região do visível, em aproximadamente $520 \quad \mathrm{~nm} \quad \mathrm{e}$ consequentemente maior eficiência fotoeletrocatalítica.

Em contra partida, o $\mathrm{BiVO}_{4}$ apresenta alguns limitadores na aplicação como material fotoeletroquímico, possuindo uma fácil recombinação do par elétron-buraco e baixa condutividade.

Neste sentido, surge a junção $\mathrm{C} \mathrm{BiVO}_{4}$, união essa que foi promovida através da carbonização do polímero utilizado na etapa de eletrofiação das nanofibras. A carbonização do PVA, fornece o carbono necessário para a união entre os aglomerados de $\mathrm{BiVO}_{4}$, formando assim fibras relativamente uniformes e ininterruptas, apresentando a junção entre ambos os materiais. 
Reportada pela primeira vez na literatura em 1987 por Rayleigh a técnica de eletrofiação possibilita a obtenção de fibras de diâmetros entre 5 a $500 \mathrm{~nm}$ a partir de um processo simples e versátil.

A eletrofiação consiste na aplicação de uma força eletrostática para deposição de nanofibras poliméricas sobre o substrato de interesse, através da injeção da solução polimérica em um campo elétrico formado entre a agulha e o coletor, Catalani (2007).

A técnica de deposição por eletrofiação tem muitas vantagens no âmbito que tange a instrumentação da técnica, sendo uma metodologia com baixo custo, fácil manuseio e simplicidade. Necessitando apenas uma fonte de alta tensão, uma bomba de infusão e um coletor aterrado.

No processo de eletrofiação, no tubo capilar (agulha) contendo a solução polimérica é conectado a uma fonte de alta tensão. Inicialmente, a solução permanece na extremidade da agulha na forma de gota, devido sua tensão superficial. Com o incremento da tensão elétrica a gota passa a tomar uma forma alongada, formando o chamado Cone de Taylor. A partir do momento no qual as forças eletrostáticas são suficientemente fortes para superar a tensão superficial da solução polimérica, o Cone de Taylor é rompido e acontece a emissão de um jato em direção ao coletor, Lin (2012). A solidificação do material acontece no trajeto deste jato, após a evaporação do solvente, propiciando assim a formação das nanofibras em forma de manta depositada no substrato coletor, Bian (2012).

Dentre inúmeras vantagens das nanofibras frente a materiais preparados a partir de técnicas de deposição comumente utilizadas, o principal benefício está no abrupto aumento na sua área superficial, Greiner (2007).

A possibilidade de obtenção de materiais nano-estruturados com área superficial extremamente elevada coloca a técnica de eletrofiação como uma poderosa ferramenta para a produção de dispositivos para aplicação em sistemas fotovoltaicos e na degradação de poluentes orgânicos, haja vista que o funcionamento destes se dá a partir da absorção da radiação solar, a qual é diretamente proporcional à área superficial do material excitado, aumentando assim a eficiência eletrocatalítica do dispositivo.

\section{METODOLOGIA}

\subsection{SÍNTESE DO SEMICONDUTOR BiVO}

$\mathrm{O}$ semicondutor $\mathrm{BiVO}_{4}$ foi sintetizado através da técnica de Síntese de Combustão em Solução (SCS), sendo utilizadas algumas adaptações dos trabalhos já reportados na literatura por Tokunaga (2001), Liu (2005), Timmaji (2011) e Jiang (2008).

Inicialmente foi preparada uma solução contendo ácido nítrico $(0,480 \mathrm{~g})$ e nitrato de bismuto (1,21 g), sendo solubilizados em $50,0 \mathrm{~mL}$ de solução ácida, com posterior ajuste para $\mathrm{pH}=7$. Solução esta que foi mistura com uma segunda solução já previamente preparada através da mistura de ácido cítrico $(0,500 \mathrm{~g})$ e metavanadato de amônio (0,300 g) em água recém-destilada sob aquecimento de $70^{\circ} \mathrm{C}$. Após a mistura a solução foi mantida por $20 \mathrm{~h}$ em estufa pré-aquecida em $80^{\circ} \mathrm{C}$.

Sendo essa solução posteriormente utilizada juntamente com solução aquosa de PVA para a formação das fibras.

Para a caracterização do material e confirmação da formação do óxido, após aquecimento em estufa o gel formado foi submetido a calcinação em mufla a $500^{\circ} \mathrm{C}$ por 1 hora.

\subsection{PREPARO DA SOLUÇÃO TRABALHO}

Para o preparo da solução trabalho, foram adicionados $2,0 \mathrm{~mL}$ da solução contendo $\mathrm{BiVO}_{4}, 0,30 \mathrm{~g}$ de PVA e $4,0 \mathrm{~mL}$ de água sob aquecimento controlado em $75^{\circ} \mathrm{C}$ e agitação magnética para total solubilização do polímero. Após a solubilização completa, obteve-se uma solução verde viscosa, denominada solução trabalho.

\subsection{ELETROFIAÇÃO DO PVA/BiVO}

Para suporte das fibras foram preparadas lâminas do substrato condutor de 
óxido de estanho dopado com flúor (FTO), previamente lavadas e com área superficial controlada $\left(1 \mathrm{~cm}^{2}\right)$.

O processo de eletrofiação foi realizado com o auxílio de uma fonte de alta tensão 0-30 kV, uma bomba de infusão para seringa e um coletor rotativo contendo um substrato de FTO.

Utilizou-se uma seringa de $3,0 \mathrm{~mL}$, com agulha de $0,55 \times 20 \mathrm{~mm}$, vazão de 0,1 $\mathrm{mL} / \mathrm{h}$, conectada a fonte de alta tensão com diferença de potencial mantida em 20 kV. Por fim, o coletor foi ajustado a uma rotação de 300 rpm numa distância entre o coletor e a seringa de $17 \mathrm{~cm}$, variando o tempo de eletrofiação (1h a 3h) por etapa.

\subsection{CARACTERIZAÇÃO DOS MATERIAIS}

\subsubsection{DIFRATOMETRIA DE RAIOS $X$}

As medidas foram feitas no Laboratório de Materiais Cerâmicos - LaMaC, do Departamento de Física, da Faculdade de Ciências e Tecnologia da UNESP, Campus de Presidente Prudente. Foi usado um equipamento marca Shimadzu, modelo XRD6000 , usando radiação $\mathrm{Cu} \mathrm{K}_{a 1}(\lambda=1,5406 \AA)$ e $\mathrm{Cu} \mathrm{K}_{\alpha 2}(\lambda=1,5444 \AA)$, tensão de $40 \mathrm{kV}$ e corrente de $30 \mathrm{~mA}$. A varredura foi feita num intervalo de ângulos $2 \theta$ de $05^{\circ}$ a $50^{\circ}$, usando fendas de divergência de $1^{\circ}$ e de recepção de $1^{\circ}$, velocidade de varredura de $2^{\circ} / \mathrm{min}$. Os picos de difração de cada composto foram indexados usando as fichas cristalográficas do banco de dados JCPDS-ICDD (Joint Committee on Powder Diffraction Standards - International Center for Diffraction Data) disponíveis no software Crystallographica Search-Match versão 2.1.

\subsubsection{ANÁLISE TÉRMICA (DSC-TGA)}

A análise térmica foi realizada no Laboratório de Materiais Cerâmicos - LaMaC, do Departamento de Física, da Faculdade de Ciências e Tecnologia da UNESP, Campus de Presidente Prudente. $O$ equipamento utilizado foi 0 aparelho da marca $T A$ Instruments - Brasil, modelo SDT Q600. A análise térmica é utilizada para identificar as reações ocorridas no material estudado em decorrência da variação de temperatura.

Foram realizados testes com o pó sintetizado e calcinado e com uma amostra da solução de trabalho contendo o polímero $\mathrm{PVA}_{\mathrm{e}} \mathrm{BiVO}_{4}$ não calcinado. As amostras foram submetidas a temperaturas de 30 a $1100^{\circ} \mathrm{C}$, com velocidade de aquecimento de $10^{\circ} \mathrm{C} / \mathrm{min}$.

\subsubsection{CARACTERIZAÇÃO MORFOLÓGICAS}

Para a caracterização morfológica das fibras eletrofiadas foram realizadas medidas de microscopia óptica para todos os parâmetros variados, devido ao grande número de análises neste trabalho serão expostas apenas as imagens para os melhores parâmetros encontrados.

Após a otimização dos parâmetros foi realizada a microscopia eletrônica de varredura (MEV), em parceria com o Centro de Desenvolvimento de Materiais Funcionais (CDMF/CEPID/FAPESP), junto ao Laboratório Interdisciplinar de Eletroquímica e Cerâmica (LIEC). As análises foram realizadas em um equipamento de marca Zeiss, modelo Supra 35 , operando de 2 a $30 \mathrm{kV}$, equipado com filamento de emissão por campo (FEG), para a realização da MEV as nanofibras foram depositadas sobre substrato de FTO e fixadas com tinta condutora de prata ao porta amostra. Tais análises foram realizadas com a finalidade de observar a morfologia das fibras e estimar seu diâmetro e observar a homogeneidade.

\section{RESULTADOS E DISCUSSÃO}

\subsection{CARACTERIZAÇÃO DOS MATERIAIS}

A síntese do $\mathrm{BiVO}_{4}$ foi confirmada a partir das seguintes caracterizações: Difratometria de Raios-X (DRX) e Análise Térmica Diferencial-Termogravimetria (DSCTGA), conforme detalhado nos materiais e métodos.

\subsubsection{DIFRATOMETRIA DE RAIOS $X$}

Foi realizada a difratometria de raios$x$ para a comprovação da formação do $\mathrm{m}$ $\mathrm{BiVO}_{4}$ após a síntese e calcinação a $500^{\circ} \mathrm{C}$. 
O difratograma apresentado na Figura 2 é correspondente ao pó de $\mathrm{BiVO}_{4}$ e a ficha cristalográfica PDF\#41-81.

Figura 2. Difratograma do óxido $\mathrm{BiVO}_{4}$ na forma de pó obtido após $1 \mathrm{~h}$ de calcinação a $500^{\circ} \mathrm{C}$ e a ficha cristalográfica correspondente.

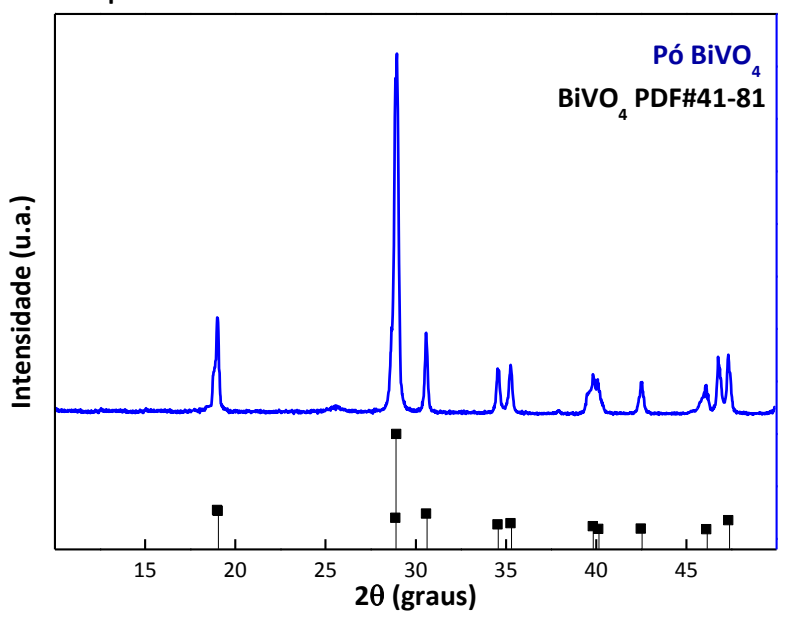

De acordo com a figura acima foi possível observar uma coerência na comparação com a ficha cristalográfica (PDF\#41-81), confirmando o êxito no método de síntese utilizado, devido a semelhança nos picos de maiores intensidades entre a amostra e a ficha.

O material sintetizado apresentou uma boa cristalinidade. Fato observado devido a estabilidade de linha base e o formato pontiagudo dos picos.

\subsubsection{ANÁLISE TÉRMICA (DSC-TGA)}

As medidas de análises térmicas diferenciais e termogravimetrias, apresentadas na Figura 3, foram realizadas com o intuito da observância do comportamento térmico das amostras. Foram utilizadas amostras do pó de $\mathrm{BiVO}_{4}$ calcinado e da solução trabalho contendo $\left(\mathrm{PVA} / \mathrm{BiVO}_{4}\right)_{\mathrm{aq}}$.
Figura 3. Termograma e curva DTA de: (A) Pó $\mathrm{BiVO}_{4}$ obtido após 1 hora de calcinação a $500^{\circ} \mathrm{C}$ e (B) Solução Trabalho (PVA/BiVO $\left.{ }_{4}\right)$.

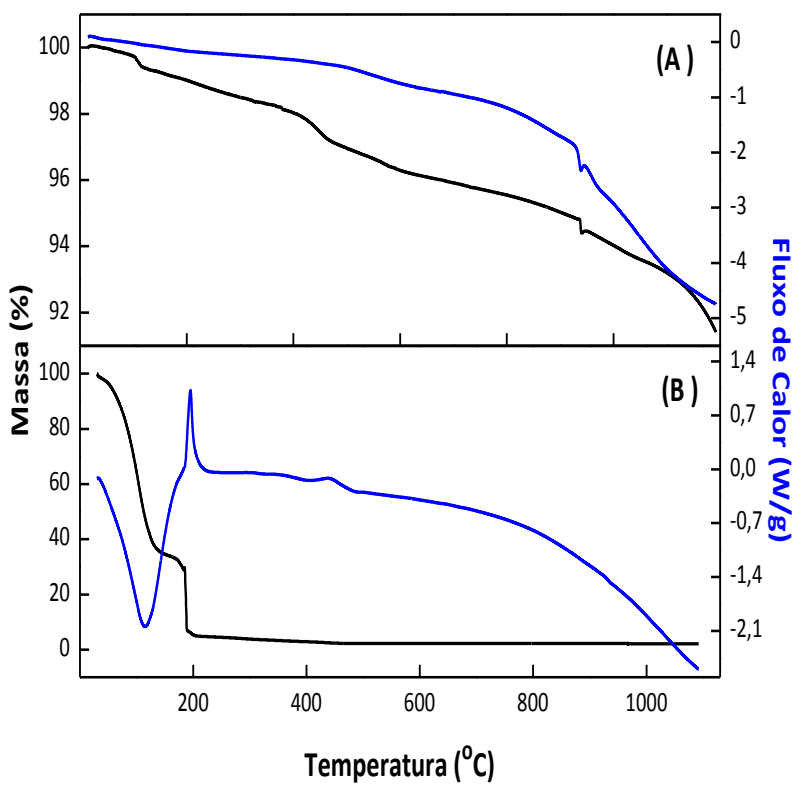

Com base na análise térmica referente ao óxido calcinado (A) pode ser observado a perda de massa de água adsorvida na amostra até aproximadamente $150^{\circ} \mathrm{C}$, seguida de uma brusca perda de massa em aproximadamente $950^{\circ} \mathrm{C}$ associada ao ponto de fusão do material analisado.

De acordo com a significativa estabilidade térmica apresentada foi possível a comprovação da irreversibilidade da forma monoclínica até a temperatura máxima obtida $\left(1100^{\circ} \mathrm{C}\right)$.

No termograma apresentado na Figura 3B, foi possível a comprovação da carbonização do polímero contido na solução de trabalho, sendo observado um pico exotérmico no fluxo de calor em aproximadamente $180^{\circ} \mathrm{C}$, temperatura compatível com o valor teórico do ponto de ebulição do PVA.

Também foi possível observar a perda de massa do sistema, inicialmente devido a água adsorvida $\left(30-150^{\circ} \mathrm{C}\right)$, seguida perda de massa pela queima do PVA $\left(\sim 180^{\circ} \mathrm{C}\right)$ e uma pequena perda até $500^{\circ} \mathrm{C}$, esta atribuída ao arranjo da forma $\mathrm{m}-\mathrm{BiVO}_{4}$.

\subsection{ELETROFIAÇÃO DO PVA/BiVO}

Para a otimização dos parâmetros de eletrofiação do $\mathrm{BiVO}_{4}$ foi utilizado o polímero 
PVA e a solução trabalho de $\mathrm{BiVO}_{4}$ em duas proporções, a primeira proporção foi $0,30 \mathrm{~g}$ de PVA e $3,0 \mathrm{~mL}$ da solução trabalho, completando o volume até $5,0 \mathrm{~mL}$ total, a segunda foi $0,30 \mathrm{~g}$ de PVA para $2,0 \mathrm{~mL}$ da solução trabalho também completando para $6,0 \mathrm{~mL}$ da solução total.

Desta forma, foi possível observar que com o passar o tempo da eletrofiação a solução contida na seringa quando adicionada $3,0 \mathrm{~mL}$ da solução trabalho iniciava um processo de cristalização, dificultando assim o processo. Portanto, utilizou-se para as demais $0,30 \mathrm{~g}$ do PVA para $2,0 \mathrm{~mL}$ da solução de trabalho contendo $\mathrm{BiVO}_{4}$ e diluídos à um volume total de $6 \mathrm{~mL}$ com água deionizada.

Após o estudo de cada parâmetro do processo de eletrofiação foram realizadas imagens ópticas para observar a formação das fibras. Desta forma, os seguintes parâmetros foram fixados: volume da seringa de $3,0 \mathrm{~mL}$, com agulha de $0,55 \times 20 \mathrm{~mm}$, vazão de $0,1 \mathrm{~mL} / \mathrm{h}$, conectada a fonte de alta tensão com diferença de potencial mantida em $20 \mathrm{kV}$, rotação do coletor de $300 \mathrm{rpm}$ numa distância entre o coletor e a seringa de $17 \mathrm{~cm}$ e no estudo do tempo de eletrofiação, o valor fixado foi de 3 horas, apresentando uma excelente densidade de nanofibras formadas.

\subsection{CARACTERIZAÇÃO MORFOLÓGICAS}

Para a caracterização morfológica das fibras eletrofiadas foram realizadas medidas de microscopia óptica para todos os parâmetros variados, devido ao grande número de análises neste trabalho serão expostas apenas as imagens para os melhores parâmetros dentre os estudados.

As imagens de microscopia óptica revelaram uma excelente formação das fibras sobre o substrato, apresentando homogeneidade e uma boa densidade de fibras formadas (Figura 4a). Após ampliação de 40x no microscópio foi possível analisar a dimensão do diâmetro das fibras (Figura 4b).
Figura 4. Imagens de Microscopia Óptica para fibras formadas com os parâmetros otimizados, sendo: 4A- ampliação de $10 x$ e 4B- ampliação de 100x.

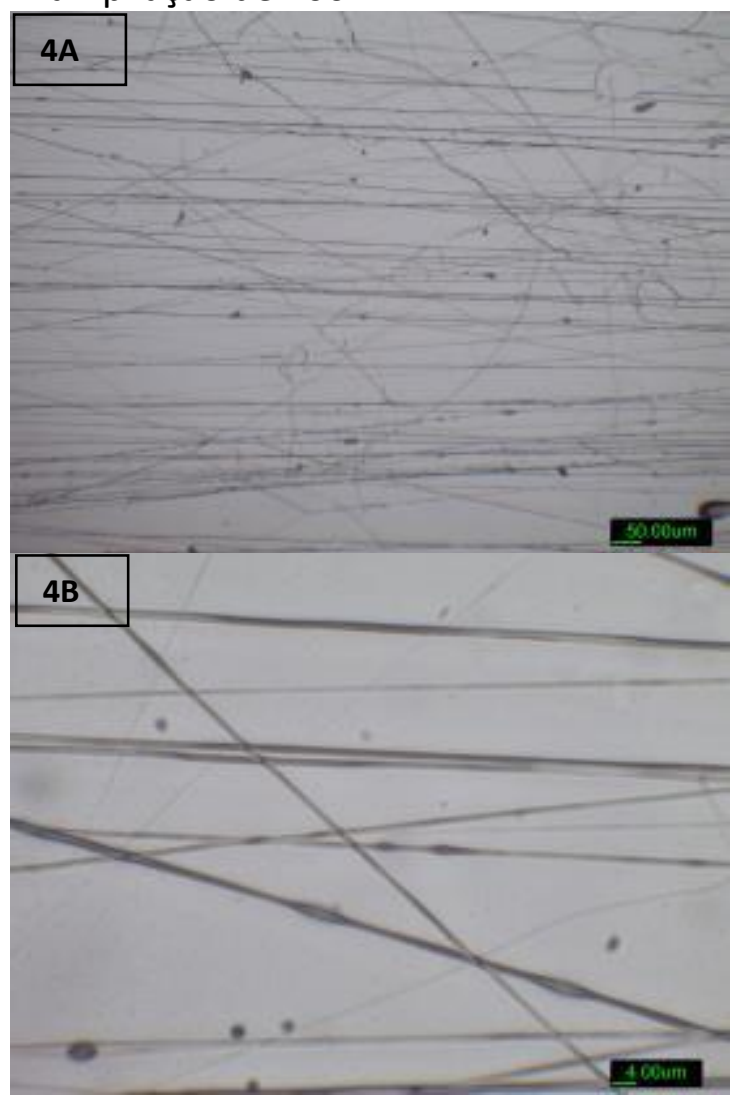

A microscopia óptica apresentada acima mostra a eficiência do processo de eletrofiação na produção de uma rede de fibras com diâmetros em escala manométrica, homogeneidade e sem apresentar coalescência, as quais causaria a diminuição da área superficial.

$\mathrm{Na}$ imagem ampliada $10 \mathrm{x}$ as fibras apresentam uma maior organização horizontal, devido ao modo de fixação da lâmina ao rolo coletor, porém quando se observa a ampliação de 100x podemos notar fibras entrelaçadas, evidenciando a desorganização das fibras, fazendo assim com que todas as fibras toquem umas as outras, ampliando a condução.

$\mathrm{Na}$ imagem de ampliação de 100x foi possível confirmar os parâmetros ótimos, devido ao fato de apresentarem fibras com diâmetros em escala abaixo de micrometro, além de não apresentar coalescência.

$\mathrm{Na}$ microscopia eletrônica de varredura (MEV), foram submetidas às 
melhores amostras encontradas na microscopia óptica, apresentando as imagens $5 A$ e $5 B$.

Figura 5. Imagens de Microscopia Eletrônica Varredura para fibras de $\mathrm{BiVO}_{4}$ formadas com os parâmetros otimizados, sendo: $5 \mathrm{~A}$ ampliação de 5.000x e 5B- ampliação de 50.000x.

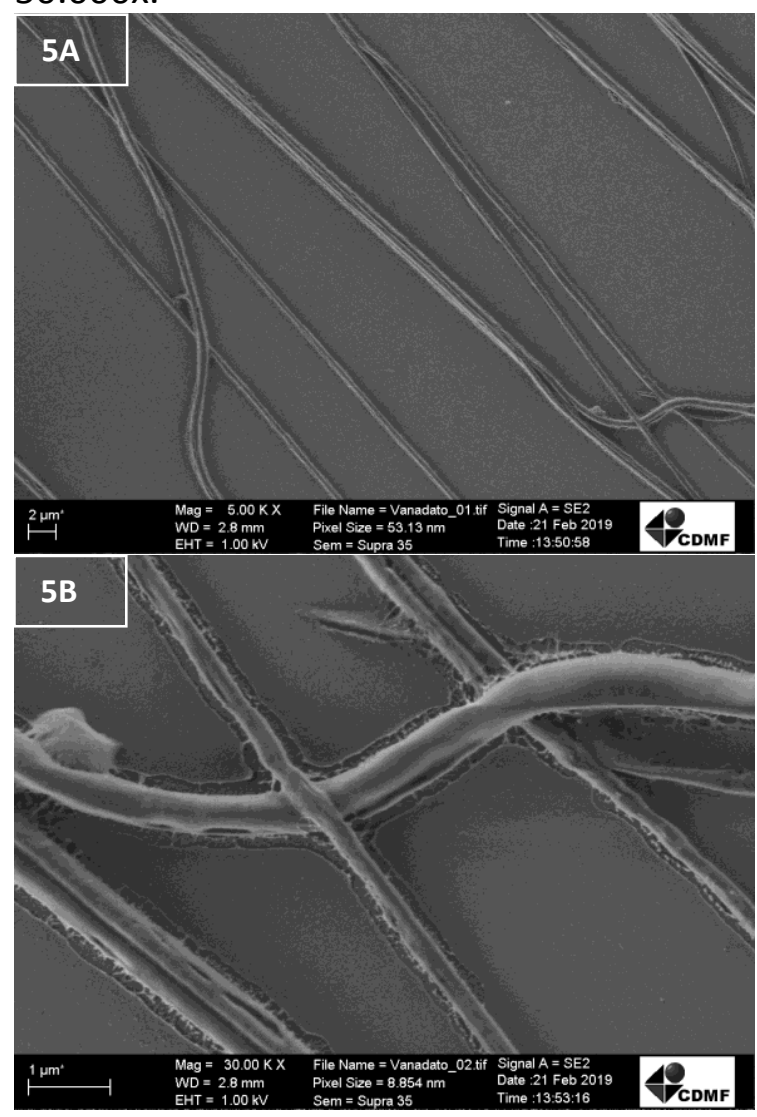

A Figura 5A apresenta uma visão panorâmica das fibras formadas mostrando uma solidez do material sem a presença de coalescência e uma boa uniformidade de capilar. Na Figura 5B, uma imagem mais aproximada, foi possível observar a perfeita formação das fibras entrelaçadas, onde apresenta a mesma fibra em sentido horizontal entrelaçando duas fibras no sentido vertical, sem que as mesmas se fundissem, causando a coalescência e diminuindo a área superficial.

Corroborando assim com a escolha dos parâmetros ótimos da eletrofiação, haja vista que as nanofibras se solidificaram no caminho até o coletor e apresentaram uma boa aderência ao substrato.
Pode-se estimar a diâmetro das fibras formadas por eletrofiação do $\mathrm{BiVO}_{4}$, conforme apresentado na Figura 6.

Figura 6. Imagem de Microscopia Eletrônica Varredura com estimativa de diâmetro para fibras de $\mathrm{BiVO}_{4}$ formadas com os parâmetros otimizados.

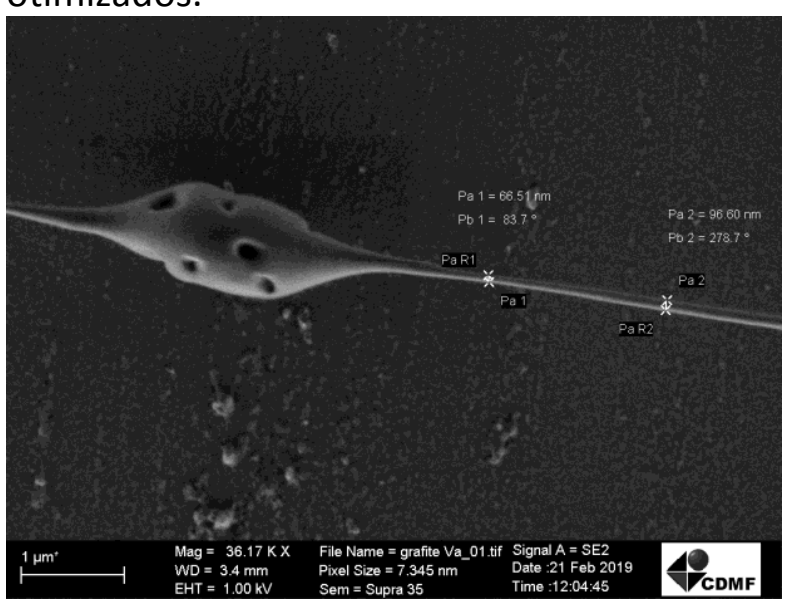

De acordo com a estimativa realizada em MEV, foi possível observar um diâmetro variando entre $66,51 \mathrm{~nm}$ e $96,60 \mathrm{~nm}$ durante a fibra, demonstrando que os parâmetros utilizados foram eficientes para formação de fibras com dimensões nanométricas.

Conforme Figura 6, podemos ainda observar um pequeno aglomerado no meio da fibra, local onde se encontra uma alta densidade do material de trabalho. Este aglomerado pode diminuir a eficiência das fibras, entretanto, a presença de interstícios no mesmo, minimiza tal complicação.

\section{CONSIDERAÇÕES FINAIS}

De acordo com os estudos realizados foi possível concluir com propriedade que a síntese do $\mathrm{BiVO}_{4}$ foi efetuada com êxito, apresentando na Difratometria de Raios- $X$ picos de difração correspondentes aos picos da ficha catalográfica.

$\mathrm{Na}$ análise térmica termogravimétrica o material apresentou grande estabilidade térmica durante a temperatura estuda sem ocorrer aparecimento de outras fases cristalinas. A análise térmica da solução trabalho confirmou a queima do polímero PVA utilizado na fabricação das nanofibras. 
No âmbito que tange os parâmetros de eletrofiação, o estudo de todas as variáveis foi realizado e fixado os melhores parâmetros, conforme descrito anteriormente. Desta forma, foram obtidas fibras com grande estabilidade térmica, com diâmetros na escala nanométricas e significativa uniformidade.

As imagens microscópicas ópticas e eletrônicas corroboraram os resultados obtidos após a fixação dos parâmetros de formação das fibras, e mostraram que tal material tem um grande potencial a ser estudado, devido ao fácil preparo dos materiais e grande aumento na área superficial das fibras formadas.

\section{AGRADECIMENTOS}

CNPq (159615/2018-6)

CDMF (CEPID/FAPESP) 2013/07296-2

\section{REFERÊNCIAS}

BIAN, S. S.; JAYARAM, S.CHERNEY, E. A. Electrospinning as a New Method of Preparing Nanofilled Silicone Rubber Composites. IEEE Transactions on Dielectrics and Electrical Insulation, v. 19, n. 3, p. 777785, 2012.

https://doi.org/10.1109/TDEl.2012.6215080

CATALANI, L. H.; COLLINS, G.JAFFE, M. Evidence for molecular orientation and residual charge in the electrospinning of poly(butylene terephthalate) nanofibers. Macromolecules, v. 40, n. 5, p. 1693-1697, 2007. https://doi.org/10.1021/ma061342d

FUJISHIMA, A.; HONDA, K. Electrochemical Photolysis of Water at a Semiconductor Electrode. Nature, v. 238, n. 5358, p. 37-+, 1972. https://doi.org/10.1038/238037a0

GREINER, A.WENDORFF, J. H. Electrospinning: A fascinating method for the preparation of ultrathin fibres. Angewandte ChemieInternational Edition, v. 46 , n. 30, p. $5670-$ 5703, 2007.
JIANG, H. Q.; ENDO, H.; NATORI, H.; NAGAI, M.KOBAYASHI, K. Fabrication and photoactivities of spherical-shaped $\mathrm{BiVO}_{4}$ photocatalysts through solution combustion synthesis method. Journal of the European Ceramic Society, v. 28, n. 15, p. 2955-2962, 2008.

https://doi.org/10.1016/j.jeurceramsoc.2008.05. $\underline{002}$

KUDO, A.; UEDA, K.; KATO, H.MIKAMI, I. Photocatalytic $\mathrm{O}_{2}$ evolution under visible light irradiation on $\mathrm{BiVO}_{4}$ in aqueous $\mathrm{AgNO}_{3}$ solution. Catalysis Letters, v. 53, n. 3-4, p. 229-230, 1998. https://doi.org/10.1023/A:1019034728816

LIN, Q. Q.; LI, Y.YANG, M. J. Polyaniline nanofiber humidity sensor prepared by electrospinning. Sensors and Actuators BChemical, v. 161, n. 1, p. 967-972, 2012. https://doi.org/10.1016/..snb.2011.11.074

LIU, M.SUZUKI, Y. BiVO 4 Hollow Nanoplates with Improved Photocatalytic Water Oxidation Efficiency. Current Nanoscience, v. 11, n. 4, p. 499-503, 2015. https://doi.org/10.2174/1573413711666150202 $\underline{233306}$

LIU, H. M.; NAKAMURA, R.NAKATO, Y. Promoted photo-oxidation reactivity of particulate $\mathrm{BiVO}_{4}$ photocatalyst prepared by a photoassisted sol-gel method. Journal of the Electrochemical Society, v. 152, n. 11, p. G856-G861, 2005. https://doi.org/10.1149/1.2051868

PARK, Y.; MCDONALD, K. J.CHOI, K. S. Progress in bismuth vanadate photoanodes for use in solar water oxidation. Chemical Society Reviews, v. 42, n. 6, p. 2321-2337, 2013. https://doi.org/10.1039/C2CS35260E

TOKUNAGA, S.; KATO, H.KUDO, A. Selective preparation of monoclinic and tetragonal $\mathrm{BiVO}_{4}$ with scheelite structure and their photocatalytic properties. Chemistry of Materials, v. 13, n. 12, p. 4624-4628, 2001. https://doi.org/10.1021/cm0103390 
TIMMAJI, H. K.; CHANMANEE, W.; DE TACCONI, N. R.RAJESHWAR, K. Solution Combustion Synthesis of $\mathrm{BiVO}_{4}$ Nanoparticles: Effect of Combustion Precursors on the Photocatalytic Activity. Journal of Advanced Oxidation Technologies, v. 14, n. 1, p. 93-105, 2011. https://doi.org/10.1515/jaots-2011-0112

YANG, G. D.; JIANG, Z.; SHI, H. H.; XIAO, T. C.YAN, Z. F. Preparation of highly visible-light active $\mathrm{N}$-doped $\mathrm{TiO}_{2}$ photocatalyst. Journal of Materials Chemistry, v. 20, n. 25, p. 53015309, 2010. https://doi.org/10.1039/c0jm00376j 